\title{
THE EFFECT OF LEARNING ON EXPERIMENTAL EVOLUTION OF RESOURCE PREFERENCE IN DROSOPHILA MELANOGASTER
}

\author{
Frederic Mery ${ }^{1}$ And TAdeusz J. KaweCKI ${ }^{2}$ \\ Division of Ecology and Evolution, Department of Biology, University of Fribourg, Chemin du Musée 10, \\ CH-1700 Fribourg, Switzerland \\ ${ }^{1}$ E-mail: frederic.mery@unifr.ch \\ ${ }^{2}$ E-mail: tadeusz.kawecki@unifr.ch
}

\begin{abstract}
Learning is thought to be adaptive in variable environments, whereas constant, predictable environments are supposed to favor unconditional, genetically fixed responses. A dichotomous view of behavior as either learned or innate ignores a potential evolutionary interaction between the learned and innate components of a behavioral response. We addressed this interaction in the context of oviposition substrate choice in Drosophila melanogaster, asking two main questions. First, will learning also evolve in a constant environment in which it always pays to show the same choice? Second, how does an opportunity to learn affect the evolution of the innate (genetic) component of oviposition substrate choice? We exposed experimental populations to four selection regimes, involving selection on oviposition substrate preference (an orange versus a pineapple medium). In two selection regimes the flies were selected for preference either for the orange medium, or for the pineapple medium. In the remaining two selection regimes the flies were also selected for preference for either orange or pineapple, but additionally could use past experience (aversion learning) to decide which medium it paid to avoid. Lines exposed to the latter selection regimes evolved improved learning ability, indicating that learning may be advantageous even if the same behavioral response is favored every generation. Furthermore, of the two selection regimes that favored oviposition on the pineapple medium, the regime that allowed for learning led to the evolution of a stronger innate preference for pineapple, than the regime that did not allow for learning. In contrast, of the two regimes that selected for oviposition on the orange medium, the one that allowed for learning led to a smaller evolutionary change of the innate preference. Thus, an opportunity to learn facilitated the evolution of innate preference under selection for preference for pineapple, but hindered it under selection for preference for orange. We discuss possible mechanisms for this effect.
\end{abstract}

Key words._Baldwin effect, behavior, habitat choice, learning, plasticity, resource preference.

Received September 17, 2003. Accepted November 13, 2003.

Behavioral responses of an animal reflect an interplay between an innate and a learned component (by innate we mean the heritable component, which determines the behavior of a naive individual). The innate, genetic component reflects the evolutionary history of the population. The learned component reflects experience accumulated within the individual's lifetime. The ability to modify behavior based on experience (i.e., learning ability) is itself a product of evolution, with notable genetic differences between related species or even conspecific populations (Gould-Beierle and Kamil 1998; Girvan and Braithwaite 1998; Jackson and Carter 2001). It is usually argued that learning is advantageous in variable environments, that is, when the fitness consequences of a given behavioral action change from generation to generation, or even within an individual's life span (for reviews see Johnston 1982; Stephens 1991; Dukas 1998). (Obviously, for learning to be useful the environment must not change too fast relative to how fast the animal can learn; Dukas 1998.) On the other hand, gaining experience is often costly and error-prone (Laverty and Plowright 1988; Sullivan 1988). The energy spent on information processing and the maintenance of underlying structures may also entail fitness costs (for reviews see Johnston 1982; Mayley 1996). Finally, alleles improving learning ability may have deleterious pleiotropic effects on other fitness-related traits (Mery and Kawecki 2003). Hence, innate, ready-to-use behavioral responses are expected to be favored in stable environments, in which the same behavioral response is always optimal (Dukas 1998).

A dichotomous view of behavior as either mostly learned or mostly innate ignores potential interactions between learning and the evolution of the genetic (innate) component. This interaction is the focus of this paper. We pose two questions. First, will learning ability also be selected in a stable environment, where the same behavioral response is always favored? Second, does learning ability affect the evolution of the innate component? We address these questions using experimentally evolving Drosophila melanogaster populations as the model system, concentrating on oviposition substrate choice (or more generally diet choice) as the focal behavior.

The above two questions have been addressed with verbal arguments, and mathematical and computer models. Papaj (1994) studied a model in which genetic variation existed for both the innate component of the response and for learning ability, and the animal could get feedback from the environment about the fitness consequences of its previous behavioral actions (i.e., reinforcement). Results of that model suggest that directional selection on a behavioral response should favor the evolution of improved learning ability as long as the innate response has not reached an optimum. This prediction contradicts other models and verbal arguments (Johnston 1982; Stephens 1991; Ancel 1999); it has not been empirically tested.

Models addressing the second question led to contradictory predictions. On the one hand, learning may allow an individual to behave optimally irrespective of its genotypic value for the innate component of behavior. Thus, the ability to learn (partially) decouples the behavioral phenotype from the genotype. It has been proposed that this effect should make natural selection on the innate, heritable component of the 
behavioral response less effective, slowing down its evolution (Papaj 1994; Anderson 1995; Robinson and Dukas 1999; Ancel 2000). In contrast, a simulation model by Hinton and Nowlan (1987; see also Maynard Smith 1987) suggests that in a novel environment learning may accelerate the evolution of the innate component towards the optimum. A similar prediction has been obtained in artificial intelligence models (Belew 1989; Ackley and Littman 1991; French and Messinger 1994; Mayley 1996). These models provide some formal underpinning for the old (and vague) verbal arguments that learning (or more generally phenotypic plasticity) may accelerate evolution by allowing the population to explore greater "phenotypic space," an idea known as the Baldwin effect (Baldwin 1896; Morgan 1896; Osborn 1896). This idea is conceptually related to genetic assimilation and received renewed interest from evolutionary biologists in the context of the role of phenotype in evolution (for review see Pigliucci and Murren 2003). Although some models (French and Messinger 1994; Mayley 1996, Ancel 2000) predicted that learning may both accelerate and slow down evolution depending on the assumptions about the constraints on learning and fitness, at the empirical level the controversy remains unresolved: these contradictory predictions have not been addressed experimentally.

In order to address these questions, we exposed experimental populations of Drosophila melanogaster to selection on both innate and learned components of oviposition substrate choice. We chose this behavioral trait for several reasons. First, in most insects oviposition site preference has a direct impact on fitness. Second, genetic variation for this trait has been demonstrated in many species (reviewed by Fox 1993). Third, Drosophila lay eggs in small batches throughout their adult life. The oviposition behavior is therefore performed repeatedly during the life of an individual, and each act of oviposition site choice has a small effect on fitness. Fourth, the adults feed on the oviposition substrates, which gives them a possibility to assess the substrate quality, and thus the likely consequences of the choice for offspring fitness. These characteristics make it possible for a learned component of oviposition behavior to contribute to fitness (McNeely and Singer 2001). We have shown that, exposed to conditions that favor learning, D. melanogaster easily evolve improved learning ability for oviposition substrate preference (Mery and Kawecki 2002). In the experiment described here we applied a similar approach to study the evolutionary interplay between the innate and learned component of the choice between two oviposition media, orange and pineapple.

We exposed experimental fly populations to four selection regimes, all involving the choice between these two oviposition media. In the Innate Orange regime the flies were selected for innate (unconditioned) preference for the orange medium. The second selection regime, Learning Orange, also favored oviposition on the orange medium, but the flies were additionally previously conditioned to avoid the other (i.e., pineapple) medium. The conditioning involved exposing the flies to the pineapple medium supplemented with an aversive chemical reinforcer (quinine); selection on oviposition site preference took place three to six hours later. The other pair of selection regimes (respectively, Innate Pineapple and
Learning Pineapple) was analogous, but they selected for oviposition on the pineapple medium. The base population showed no detectable ability to respond to conditioning under these conditions (Mery and Kawecki 2002). We tested two hypotheses. First, based on Papaj's (1994) model we predicted that the Learning Orange and Learning Pineapple lines should evolve improved learning ability, despite the direction of selection being constant. No such response was expected in the Innate Orange and Innate Pineapple lines. Second, if an opportunity to learn affects the evolution of the innate component of preference, the unconditioned preference of lines from the corresponding "innate" and "learning'" regimes should be different.

\section{Materials And Methods}

\section{Base Population}

The base population originated from approximately 2000 D. melanogaster eggs collected in the summer of 1999 in Basel (Switzerland). The flies were subsequently maintained on a cornmeal medium at $25^{\circ} \mathrm{C}$ in complete darkness and constant humidity (70-75\%). The selection experiment began several months after eggs collection, which should have allowed the flies to adapt to the laboratory environment.

\section{Experimental Evolution}

The experiment consisted of four selection regimes and a fifth control regime, initially each with eight replicate lines. Every generation in each selection regime 150 adult flies (aged 14 days counted from egg) from each selection line were transferred to a cage $(19 \times 12 \times 13 \mathrm{~cm})$ and allowed to oviposit during three consecutive periods of three hours each, in darkness, $25^{\circ} \mathrm{C}$, and $70 \%$ relative humidity. During each period we offered the flies a choice between two oviposition substrates: an orange medium and a pineapple medium. These media were prepared from $100 \%$ orange or pineapple juice from concentrate and $6.6 \mathrm{~g} / \mathrm{l}$ of agar. At the bottom of the cage a petri dish with $10 \mathrm{ml}$ of the orange medium and another with $10 \mathrm{ml}$ of the pineapple medium, each with a drop of yeast, were attached at the ends of plastic tubes (height $5 \mathrm{~cm}$, diameter $6 \mathrm{~cm}$ ). A fresh set of petri dishes with the media was provided at the beginning of the three periods; their position was randomized. Eggs laid on each medium in each period were counted; oviposition preference was scored for each period as the proportion of eggs laid on the orange medium.

In the selection regime Learning Orange (Fig. 1a), the pineapple medium offered in the first 3-h period was supplemented with $4 \mathrm{~g} / \mathrm{l}$ of quinine hydrochloride. At this concentration quinine is strongly aversive when tasted by the flies, and is lethal to the larvae (F. Mery, unpubl. data), although several hours of exposure in the adult stage have no detectable effect on subsequent fecundity (F. Mery, unpubl. data). Quinine was not added to either medium offered in periods 2 and 3. During the first period (conditioning period) the flies of this selection regime thus had an opportunity to associate the smell or taste of pineapple with the aversive taste of quinine. Their associative (aversion) learning ability would be manifested as an increased preference for the orange 
(a) Learning Orange

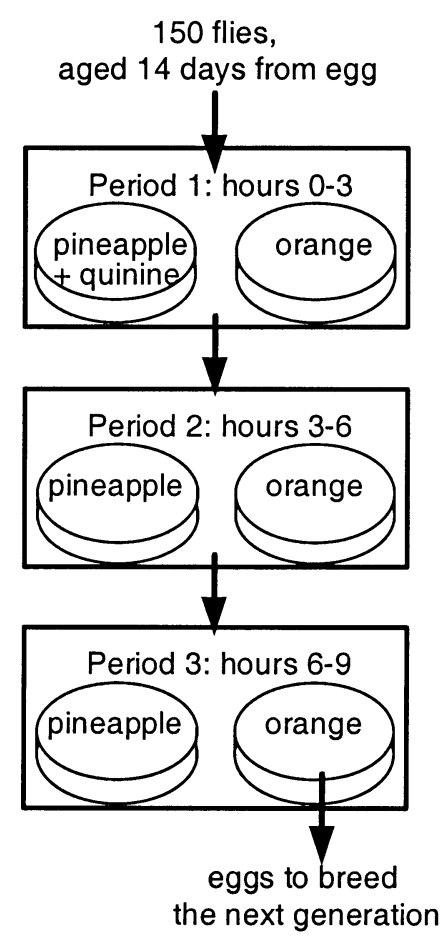

(b) Innate Orange

150 flies, aged 14 days from egg
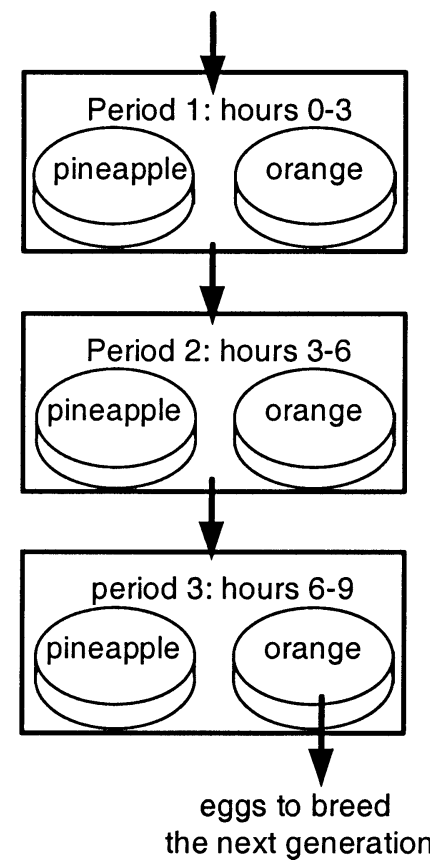

FIG. 1. The design of selection regimes. (a) Learning Orange; (b) Innate Orange. Selection regimes Learning Pineapple and Innate Pineapple followed the same design except that the eggs to breed the next generation were collected from the pineapple medium, and in Learning Pineapple the orange medium was supplemented with quinine in period 1.

medium in periods 2 and 3 (test periods). Although a simpler design with a single test period would suffice to impose selection on both learned and innate components of oviposition site preference, we used two test periods to see how the response to conditioning decays with time elapsed since the end of the conditioning period. Providing fresh media after three hours also reduced the potential influence of egg overcrowding on the female motivation to oviposit. The next generation was bred from 250 eggs laid on the orange medium in period 3; in a few cases when not enough eggs were laid in period 3, we also used eggs laid on the orange medium in period 2.

The selection regime Innate Orange was identical to Learning Orange except that quinine was never added to any medium (Fig. 1b). This regime thus imposed selection on the unconditioned preference, that is, favored flies with innate, genetically transmitted preference for orange. The other two selection regimes, Learning Pineapple and Innate Pineapple were mirror images of Learning Orange and Innate Orange, respectively. That is, in Learning Pineapple the orange medium offered in period 1 was supplemented with quinine, and in both selection regimes the next generation was bred from eggs laid in period 3 on the pineapple medium. The Control regime involved breeding the flies from eggs laid on the orange medium in the odd-numbered generations and from eggs laid on the pineapple medium in the even-numbered generations. That is, in odd-numbered generations the Con-

trol regime was identical to Innate Orange and in even-numbered generations to Innate Pineapple; quinine was never added to any medium.

In all selection regimes the eggs used to breed the next generation were rinsed with water and transferred to a 250$\mathrm{ml}$ bottle containing $21 \mathrm{ml}$ of a standard cornmeal medium. All larvae were thus always reared on the same medium, which precluded any preferences induced by larval medium. The generation time was 14 days.

These selection regimes were applied for 47 generations. One Learning Orange line was accidentally lost at generation 2 . Due to technical problems (accidental insecticide poisoning in the laboratory) at generation 27 we lost 14 of the 39 lines (four lines of Learning Pineapple, four lines of Innate Pineapple, three lines of Learning Orange, two lines of Innate Orange, and one Control line) and the population size of some other lines was temporarily reduced (in one case to only about 20 adults). To facilitate recovery, selection regimes were suspended for generations 27-31; selection was also not applied at generations 11,35 , and 44 for other reasons. At those generations flies laid eggs on a standard cornmeal medium. The mild bottleneck that resulted from the accident could have caused some loss of genetic variation, and thus potentially reduced performance due to increased homozygosity (inbreeding depression). This possibility was addressed as a part of another experiment, performed 10 generations after the accident, in which we tested for inbreeding depression in the Control lines and in another set of lines selected for improved learning. The latter lines are not described in this paper, but were maintained in parallel and similarly affected by the insecticide accident. We found no evidence of inbreeding depression either for learning ability or for larval viability under competitive conditions (Mery and Kawecki 2003). Extrapolating these results to the lines that are the focus of this paper, and noting that they show no apparent reduction in fecundity or viability, we believe that they are also unlikely to suffer greatly from inbreeding depression. Therefore we decided to continue the selection after the lines recovered, and to include in this paper data from an assay performed at generation 46; that is, 19 generations after the accident.

\section{Assay of the Innate Preference and Response to Conditioning}

To analyze the evolution of both components of the oviposition substrate preference, after 23 and 46 generations of selection, we assayed flies from each selection regime for both the innate (unconditioned) preference and its response to aversive conditioning. From each replicate selection line of each selection regime, as well as from each control line, we obtained three samples of 150 flies each (females + males, 14 days old counting from egg). The first sample was used to measure the innate (i.e., not conditioned) preference. This involved a design identical to that used in the Innate Orange and Innate Pineapple selection regimes. That is, the flies were transferred to a cage and allowed to oviposit for three consecutive 3-h periods, having a choice between the orange and the pineapple medium, neither containing quinine (Fig. 1b). The second sample was conditioned to avoid pineapple in an 
identical way as applied in the course of selection in the Learning Orange selection regime. That is, quinine was added to the pineapple medium offered in the period 1 , but not periods 2 and 3 (Fig. 1a). The third sample was conditioned in an analogous way to avoid orange. Eggs laid on each medium in periods 2 and 3 were then counted. The learned component of the oviposition substrate choice is reflected in the effect of treatment on the distribution between the media of eggs laid in periods 2 and 3.

\section{Statistical Analysis}

PROC GLM of SAS 8.02 statistical package (Littell et al. 1991) was used for all analyses; $F$-tests were based on type 3 sums of squares. The focal trait was the realized oviposition substrate preference, measured as the proportion of eggs laid on the orange versus pineapple medium. All proportions were calculated separately for periods 2 and 3 , and $\operatorname{arcsine}\left(X^{1 / 2}\right)$ transformed before the analysis (analysis of untransformed data produced virtually identical results and is not reported). Only realized preference in period 3 was the target of selection in the selection regimes (only eggs laid in period 3 were used to establish the next generation). Most of the analysis reported in detail below is therefore based on data from period 3 only. However, we also report a repeated-measures analysis of response to conditioning on data from period 2 and period 3 . This repeated-measures analysis would have more power to detect effects of conditioning and would test for changes of the response with time elapsed since the conditioning period. Such changes are expected to occur as the animals forget what they have learned. Data from period 1 were excluded from the analysis because in those treatments, in which quinine was added to one of the media offered in period 1, the distribution of eggs laid in period 1 simply reflected avoidance of quinine. This avoidance was almost complete from the first generation (less than $2 \%$ of eggs were laid on the quinine-containing medium), and is of no interest here. The results from generations 23 and 46 were analyzed separately.

\section{Innate preference}

The analysis of innate preference was based on data from the treatment involving no conditioning, which we take as an operational measure of innate preference (see Discussion). First, to see which selection regimes resulted in an evolutionary change of the innate preference, we performed a oneway ANOVA on the proportion of eggs laid on orange (in period 3), and used Dunnett's test to compare each selection regime with the Control regime.

We then addressed our main question concerning the innate preference, namely whether an opportunity to learn in the course of selection affected the evolution of innate (unconditioned) preference. To do that, we performed a two-way ANOVA, using the proportion of eggs laid on the rewarding medium (i.e., orange medium in selection regimes Innate Orange and Learning Orange; pineapple medium in Innate Pineapple and Learning Pineapple). The factors were regime type (Innate vs. Learning) and direction of selection (Orange vs. Pineapple); this analysis excluded the Control lines. The effect of regime type could depend on the direction of selection (which would be reflected in regime type $\times$ direction of selection interaction). Therefore we also tested separately for differences between selection regimes that were identical except for the opportunity for aversion learning; that is, Learning Orange versus Innate Orange, and Learning Pineapple versus Innate Pineapple. This was done with planned contrasts within the framework of the above two-way ANOVA.

\section{Response to conditioning}

The aim of this analysis was to test the hypothesis that flies from selection regimes that allowed an opportunity for learning (i.e., Learning Orange and Learning Pineapple) should show a response to conditioning: they should lay a greater proportion of eggs on orange when conditioned to avoid pineapple than when conditioned to avoid orange. Ideally, the unconditioned preference should be intermediate. This response should be absent or weaker in the other selection regimes and the Control lines.

The response to conditioning was first analyzed separately for each selection regime. We used the realized preference (arscine-transformed proportion of eggs laid on orange) from period 3 as the dependent variable in a two-way ANOVA, with treatment (not conditioned, conditioned to avoid pineapple, conditioned to avoid orange) as a fixed factor and replicate line as a random factor (block). Additionally, we performed a repeated-measures analysis on data from periods 2 and 3 , with treatment and period as factors, and replicate line as a block (subject) with respect to both factors. The treatment $\times$ period interaction would reflect potential decay of the response to conditioning with time elapsed since the end of conditioning (which took place in period 1).

We then directly tested the hypothesis that the Learning Pineapple and Learning Orange lines had evolved a stronger response to conditioning than the Innate Pineapple and Innate Orange lines. To do that, for each replicate line we calculated a learning score as the difference between the arcsine-transformed proportion of eggs laid in period 3 on orange when conditioned to avoid pineapple and when conditioned to avoid orange. We used the learning score as the dependent variable in a two-way ANOVA analogous to that used to analyze innate preference (see above), with regime type and direction of selection as factors. As for the innate preference, we used planned contrasts to compare the learning score between selection regimes Learning Orange and Innate Orange, as well as between Learning Pineapple and Innate Pineapple.

Finally, we addressed the relationship between the innate and learned components by testing whether the learning ability was correlated with the innate preference among replicate lines within the selection regimes Learning Orange and Learning Pineapple. To do this we calculated Pearson's correlation coefficient between unconditioned preference and the learning score (both based on data from period 3) among replicate lines within each selection regime.

\section{RESULTS}

\section{Changes in the course of selection}

At generation 0 the flies of all selection regimes laid in periods 2 and 3 on average $58 \%$ of their eggs on the orange 


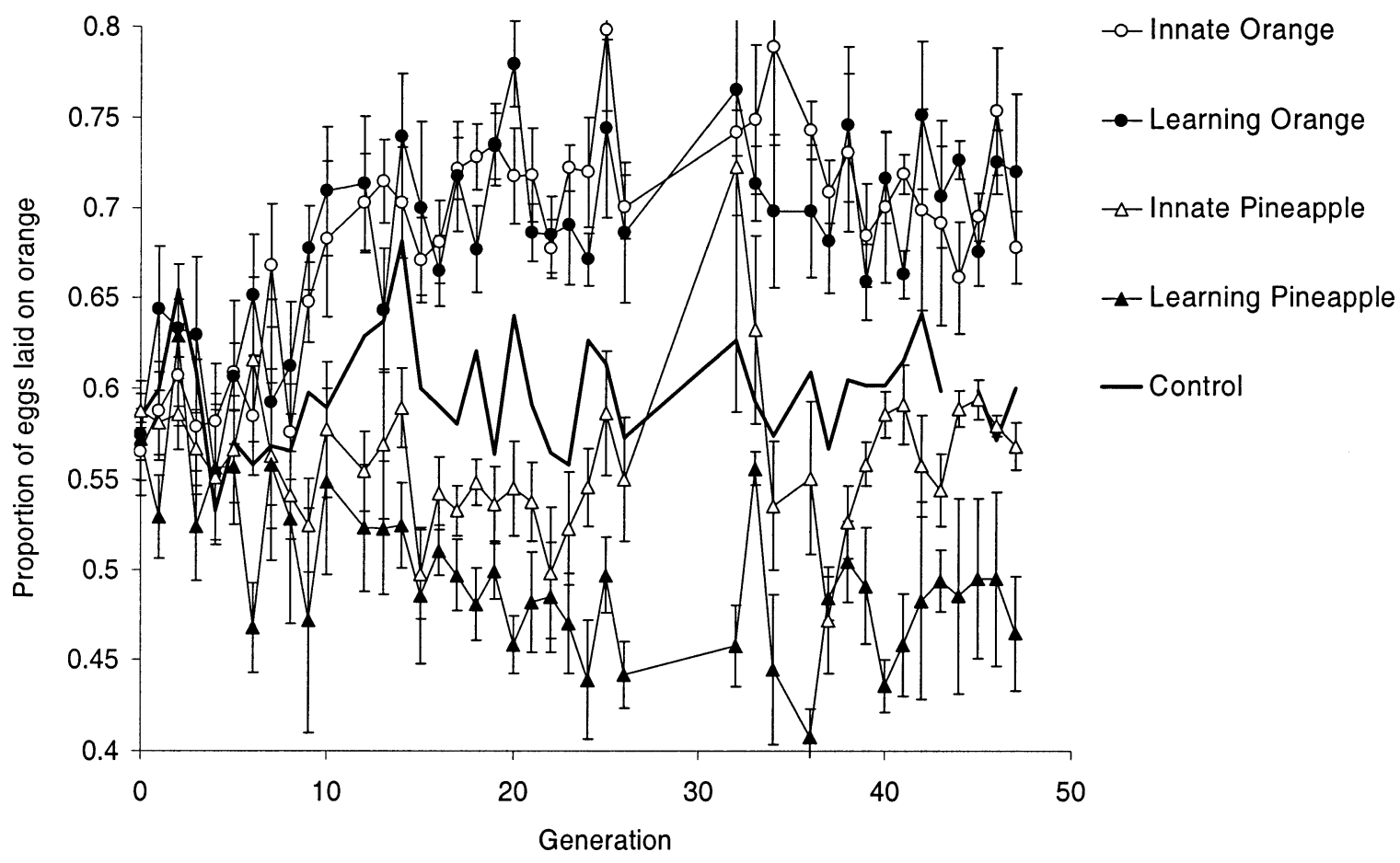

FIG. 2. Changes in the realized preference, measured as the proportion of eggs laid on the orange medium in period 3, in the course of experimental evolution. Bars represent \pm one standard error. Standard errors for the control lines were not plotted for the sake of readability. Data from generations 11 and $27-31$ are missing (see text).

medium. This proportion was significantly different from $50 \%\left(t=11.6, P<10^{-3}\right)$, indicating a slight preference for the orange medium. It did not differ among the selection regimes (ANOVA, $F_{3,28}=0.8, P=0.48$ ), suggesting no detectable response to conditioning in the base population, in agreement with our earlier results (Mery and Kawecki 2002).

The realized oviposition substrate preference changed in the course of selection in the predicted directions (Fig. 2). Until generation 27 the proportion of eggs laid on the orange medium increased in the selection regimes Learning Orange and Innate Orange, and decreased in Learning Pineapple and Innate Pineapple, although in the Innate Pineapple this decrease was much slower than in the Learning Pineapple lines. After generation 31 no systematic changes of the realized preference were apparent in the surviving lines of any selection regime (Fig. 2). The innate preference of the Control lines did not change in the course of the experiment: the proportion of eggs they laid without conditioning at generation $23(57 \%)$ and generation $46(60 \%)$ was essentially identical to that at generation $0(58 \%)$. No differences between Control populations and the base population maintained on a standard cornmeal medium were detected (Mery and Kawecki 2002).

\section{Number of eggs}

In the assays of innate preference and response to conditioning most replicates laid between 200 and 600 eggs in period 3; the average was about 380 eggs (range 105-987). The means per selection regime at generation 23 varied between 320 (Control) and 430 eggs (Innate Pineapple); at gen- eration 46 they varied between 315 (Innate Orange) and 480 (Innate Pineapple). Conditioning had no apparent effect on the number of eggs laid in period 3. In all selection regimes the flies laid around $15 \%$ fewer eggs in period 2 than in period 3. This difference probably reflects the natural tendency of fruit flies to lay more eggs in late afternoon (and therefore during period 3).

\section{Innate preference}

The proportion of eggs laid in period 3 on the orange medium in the absence of conditioning differed among the selection regimes (black bars in Fig. 3; one-way ANOVA, $F_{4,34}=24.7$ at generation $23, F_{4,21}=9.3$ at generation 46, both $P<0.001$ ), indicating a response of innate preference to selection. Dunnett's test indicated that at generation 23 Innate Orange, Learning Orange, and Learning Pineapple diverged significantly from the Control lines $(P<0.05)$. At generation 46 the power was lower because of fewer lines, and the only selection regime significantly different from the Control by Dunnett's test was Innate Orange.

More importantly from the viewpoint of our question, the opportunity to learn offered in the course of selection affected the evolution of the innate preference, but the effect had a different sign for the two directions of selection. This is reflected in Table 1 as a significant regime type $\times$ direction of selection interaction, combined with no main effect of regime type. Specifically, in the absence of conditioning, the Learning Orange lines showed a weaker preference for the rewarding medium, that is, the medium they were selected to prefer (orange) than the Innate Orange lines. In contrast, the Learning Pineapple lines showed a stronger unconditioned 


\section{Period 2}
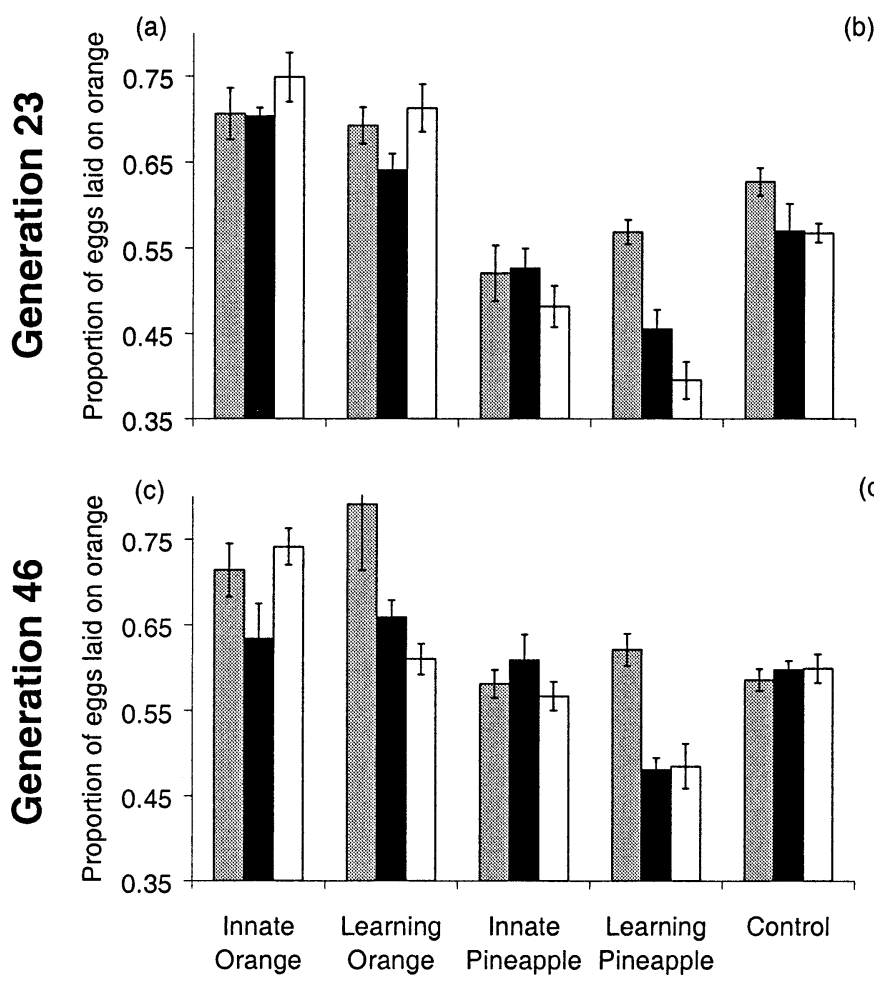

Period 3

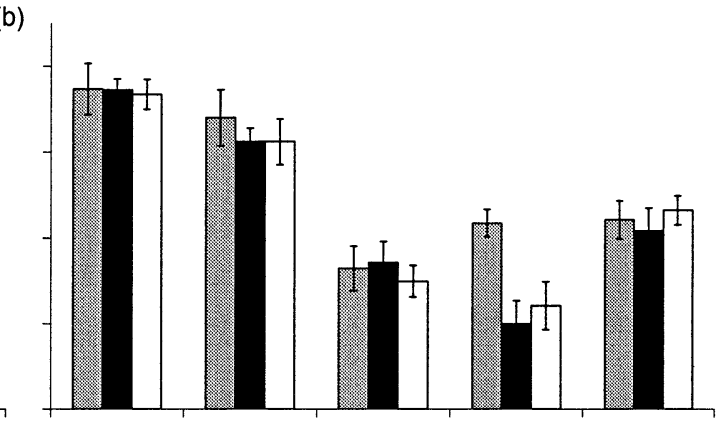

(d)

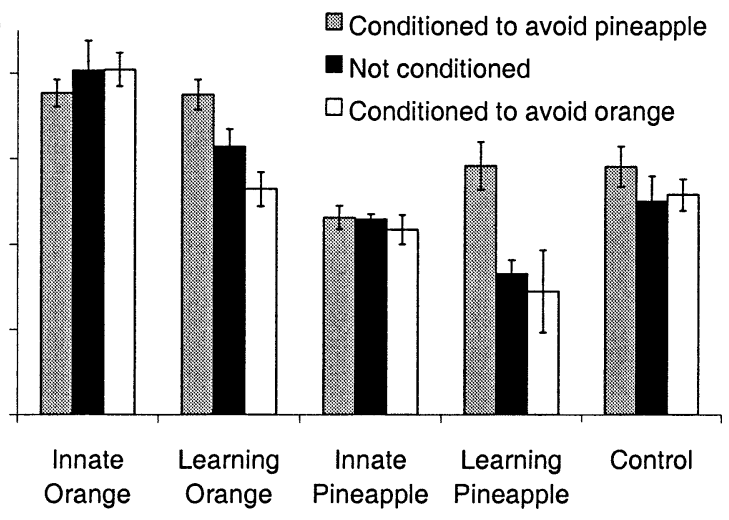

FIG. 3. The innate preference and the response to conditioning assayed at generation 23 in period 2 (a) and period 3 (b) and at generation 46 in period 2 (c) and period 3 (d). "Conditioned to avoid pineapple" means that quinine was present in the pineapple medium offered in period 1.

preference for the medium they were selected to prefer (i.e., pineapple) than the Innate Pineapple lines. These pairwise differences were consistent between the two assays (generation 23 and 46) and between period 2 and 3 (black bars in Fig. 3). They are statistically supported by the significance of the planned contrasts in Table 1. For the Innate Pineapple versus Learning Pineapple this contrast was only significant at generation 23. However, even at generation 46 all four surviving Learning Pineapple lines laid a greater proportion of eggs on pineapple $(45-52 \%)$ than any Innate Pineapple line $(41-43 \%)$. When data from both period 2 and period 3 were analyzed simultaneously in a repeated-measures ANOVA, the contrast between Innate Pineapple and Learning
Pineapple was significant also at generation $46(P=0.027$; details not reported).

\section{Response to conditioning}

Analysis of data from period 3 indicates that the Innate Orange, Innate Pineapple, and Control lines did not detectably respond to conditioning in either assay (all $P>0.35$ ). In contrast, the Learning Pineapple lines responded to conditioning both at generation $23\left(F_{2,14}=17.3, P<0.001\right)$ and at generation $46\left(F_{2,6}=5.55, P=0.04\right)$. This response was, however, strongly asymmetric: in period 3 the Learning Pineapple changed their realized preference when condi-

TABLE 1. Two-way analysis of variance on the proportion of eggs laid in the absence of conditioning on the rewarding resource, that is, on the medium the flies were selected to prefer (orange in Innate Orange and Learning Orange; pineapple in Innate Pineapple and Learning Pineapple). Regime type refers to the presence versus absence of opportunity to learn in the course of selection; direction of selection refers to selection to prefer orange versus pineapple. The analysis is based on data from period 3 only.

\begin{tabular}{lll}
\hline \hline \multicolumn{1}{c}{ Factor } & \multicolumn{1}{c}{ Generation 23} & Generation 46 \\
\hline Regime type & $F_{1,27}=0.0, P=0.88$ & $F_{1,15}=0.4, P=0.54$ \\
Direction of selection & $F_{1,27}=71.9, P<0.001$ & $F_{1,15}=72.4, P<0.001$ \\
Regime type $\times$ direction of selection & $F_{1,27}=10.1, P=0.0037$ & $F_{1,15}=7.2, P=0.017$ \\
Planned contrasts & & $F_{1,15}=6.4, P=0.023$ \\
Innate Orange vs. Learning Orange & $F_{1,27}=4.4, P=0.045$ & $F_{1,15}=1.8, P=0.20$ \\
Innate Pineapple vs. Learning Pineapple & $F_{1,27}=5.8, P=0.024$ & \\
\hline
\end{tabular}


LEARNING AND EVOLUTION

TABLE 2. Repeated-measures analysis of variance on the proportion of eggs laid on the orange medium with and without conditioning (assays at generation 23 and 46). The analysis has been done separately for the two assays and for each selection regime.

\begin{tabular}{|c|c|c|c|c|c|}
\hline Factor & Innate Orange & Innate Pineapple & Learning Orange & Learning Pineapple & Control \\
\hline \multicolumn{6}{|l|}{ Generation 23} \\
\hline Treatment & $F_{2,35}=0.48$ & $F_{2,35}=2.62 \dagger$ & $F_{2,30}=2.72$ & $F_{2,35}=55.7 * * *$ & $F_{2,35}=1.49$ \\
\hline Period & $F_{1,35}=0.00$ & $F_{1,35}=0.04$ & $F_{1,30}=0.35$ & $F_{1,35}=4.20 *$ & $F_{1,35}=1.07$ \\
\hline Treatment $\times$ period & $F_{2,35}=0.79$ & $F_{2,35}=0.38$ & $F_{2,30}=1.54$ & $F_{2,35}=5.53 * *$ & $F_{2,35}=1.48$ \\
\hline \multicolumn{6}{|l|}{ Generation 46} \\
\hline Treatment & $F_{2,25}=1.76$ & $F_{2,15}=1.19$ & $F_{2,20}=6.51 * *$ & $F_{2,15}=17.42 * * *$ & $F_{2,30}=0.29$ \\
\hline Treatment $\times$ period & $F_{2,25}=2.64 \dagger$ & $F_{2,15}=0.46$ & $F_{2,20}=1.09$ & $F_{2,15}=0.10$ & $F_{2,30}=1.09$ \\
\hline
\end{tabular}

tioned to avoid pineapple, but when conditioned to avoid orange they showed the same realized preference as in the absence of conditioning (Fig. 3). At generation 23 the response of the Learning Pineapple lines to conditioning was less asymmetric when measured in period 2; that is, immediately after conditioning, but at generation 46 the asymmetry was already apparent in period 2 (Fig. 3). The Learning Orange lines did not show a detectable response to conditioning at generation $23\left(F_{2,12}=2.62, P=0.11\right)$, but a clear response to conditioning was observed at generation $46\left(F_{2,8}\right.$ $=9.25, P=0.008)$. This response was more symmetric than that of the Learning Pineapple lines.

The loss of the response to conditioning to avoid orange between period 2 and period 3 shown by the Learning Pineapple flies was reflected in the significance of period and treatment $\times$ period interaction when data from both period 2 and period 3 were analyzed together in a repeated-measures analysis (details in Table 2). Otherwise, this analysis confirmed the significant response to conditioning of the Learning Pineapple lines at generation 23 and 46, and of the Learning Orange lines at generation 46. It also indicated a trend of the Innate Pineapple lines to respond to conditioning at generation $23(P=0.09)$; this trend was absent at generation $46(P=0.33)$.

The two-way analysis of variance on the learning score confirmed that flies from the two selection regimes involving learning (Learning Orange and Learning Pineapple) showed better learning at generation 46 compared to the two Innate selection regimes (significant effect of regime type and both planned contrasts in Table 3). At generation 23 the overall effect of regime type was not significant (Table 3), reflecting the fact that only the Learning Pineapple lines showed a response to conditioning in that assay (Fig. 3, Table 2). Even the Learning Pineapple lines had only marginally signifi- cantly better learning score than the Innate Pineapple lines (planned contrast in Table 3).

At generation 23 we observed a marginally significant positive correlation among replicate lines of the Learning Pineapple selection regime between the learning score (in period 3 ) and innate preference for pineapple (Fig. 4a). The correlation was essentially identical when calculated with data from period 2 ( $r=-0.67, P=0.07)$. It was also virtually identical when calculated with the original (untransformed) data, indicating that it is not an artifact of scale. An even stronger, although again only marginally significant, correlation between the learning score and innate preference was observed among the four surviving Learning Pineapple lines at generation 46 (Fig. 4b). Again, an analogous correlation estimated from data from period 2 was nearly identical $(r=$ $-0.89, P=0.11)$. In contrast, no clear pattern of correlation between the learning score and the innate preference (measured in period 3) was observed among the Learning Orange lines $(r=-0.06$ and $r=-0.17$ at generation 23 and 46, respectively, both $P>0.7)$. When calculated from data from period 2 these correlations tended to be slightly more negative $(-0.37$ and -0.26 , respectively), but still far from significant (both $P>0.4$ ).

\section{DisCUSSION}

By exposing populations of D. melanogaster to directional selection on oviposition site preference after giving them an opportunity to learn which medium should be avoided, we aimed to address two main questions. First, will learning ability evolve in an environment in which the same, fixed behavioral response is always favored? Second, does an opportunity for learning affect the evolution of the genetically determined, innate component? The control for both ques-

TABLE 3. Two-way analysis of variance on the learning score (data from period 3).

\begin{tabular}{lll}
\hline \hline \multicolumn{1}{c}{ Factor } & Generation 23 & Generation 46 \\
\hline Regime type & $F_{1,27}=2.8, P=0.11$ & $F_{1,15}=17.4, P<0.001$ \\
Direction of selection & $F_{1,27}=1.3, P=0.27$ & $F_{1,15}=1.3, P=0.28$ \\
Regime type $\times$ direction of selection & $F_{1,27}=0.9, P=0.35$ & $F_{1,15}=0.1, P=0.83$ \\
Planned contrasts & & $F_{1,15}=3.4, P=0.004$ \\
Innate Orange vs. Learning Orange & $F_{1,27}=0.3, P=0.62$ & $F_{1,15}=2.6, P=0.020$ \\
Innate Pineapple vs. Learning Pineapple & $F_{1,27}=3.5, P=0.072$ & \\
\hline
\end{tabular}



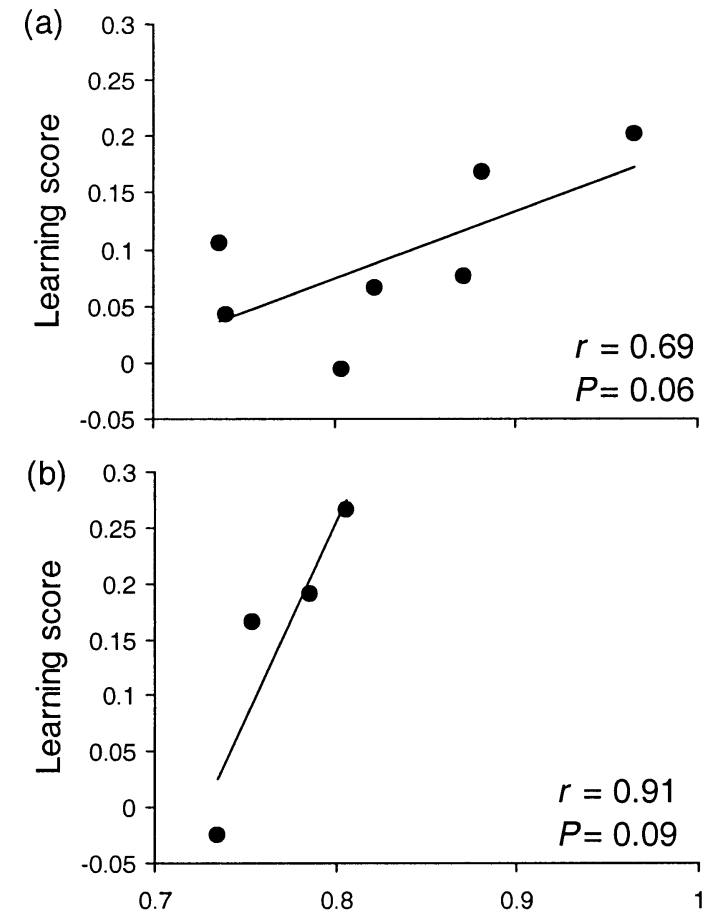

Innate preference for pineapple (transformed)

FIG. 4. The relationship between the innate preference for the rewarding (pineapple) medium and learning ability among the Learning Pineapple lines at generation 23 (a) and generation 46 (b). The learning score was calculated as the difference between the realized oviposition preference when conditioned to avoid pineapple and when conditioned to avoid orange (data from period 3). Both scales are angularly transformed. The lines are least-square regressions.

tions is provided by the regimes selecting on preference without giving the flies an opportunity to learn (Innate Orange and Innate Pineapple); we discuss the response to these selection regimes first.

\section{Evolution of Innate Preference}

We cannot exclude that even in the absence of quinine the experience with the two media in periods 1 and 2 affected subsequent preference in period 3, although this seems to be unlikely given no effect of period on the realized preference in the absence of conditioning (data not shown). Even if such an effect was present, it should not be different in lines selected in different directions: in the absence of conditioning the experience gave the flies no cue which medium it would pay to choose for oviposition. Therefore, we believe that the differences between selection regimes in the realized preference in the absence of conditioning reflect evolutionary, genetically based changes of the innate component of preference. Consequently, we use the realized preference in the absence of conditioning as an operational measure of innate preference.

Oviposition substrate preference and its evolutionary aspects have been extensively studied in herbivorous insects (Futuyma and Moreno 1988; Radtkey and Singer 1995; Thompson 1998; Nosil 2002; Scriber 2002). Although evolutionary changes of host preference in nature have been documented (Singer et al. 1993), experimental studies have often failed to obtain a response to selection on preference (Wasserman 1986; Thompson 1993). Thompson (1993; Wehling and Thompson 1997) proposed three possible mechanisms that may be responsible for evolutionary conservatism of oviposition preference: genetic constraints, weak selection, and gene flow. Our experimental design precluded gene flow. Furthermore, selection was rather strong: other things being equal, the contribution of a female fly to the next generation was proportional to the number of eggs laid on the rewarding (selected) medium. The adult population size of 150 individuals made it unlikely for drift to be able to overcome the effects of selection during the first half of the experiment (until generation 27), although drift may have played a role during the bottleneck caused by the insecticide poisoning at generation 27 . Therefore, broadly defined genetic constraints remain the most plausible explanation for the poor response of the Innate Pineapple lines to selection. However, genetic variation for a greater innate preference for the pineapple medium did exist in our base population, as the Learning Pineapple lines did evolve a stronger innate preference for this medium. Selection under the Innate Pineapple selection regime may have been opposed by possible deleterious pleiotropic effects of alleles for pineapple preference. Although we cannot exclude this possibility, it would be difficult to imagine why such pleiotropy should not affect the Learning Pineapple lines as well. Another possibility is that the greater innate preference of some individuals for pineapple may have been overridden by a tendency to oviposit where other females have oviposited. This scenario is discussed below in the subsection on the interaction between innate and learned components of preference.

\section{Evolution of Learning Ability}

In both assays (generation 23 and 46), flies from populations subject to the Learning Pineapple selection regime showed an ability to respond to conditioning. The Learning Orange flies also showed a response to conditioning at generation 46, although not at generation 23. In contrast, the Innate Pineapple, Innate Orange, and Control lines did not detectably respond to conditioning in either assay; at generation 46 the learning score of Learning Orange and Learning Pineapple lines was significantly better than that of Innate Orange and Innate Pineapple, respectively. Learning Pineapple and Learning Orange have thus evolved an improved ability for aversion learning. In contrast to the usual argument that learning is only favored in temporally varying environments (Dukas 1998), here learning ability has evolved under directional selection regimes that always favored the same response. This result supports the prediction of a model by Papaj (1994), who predicted that learning will be favored even under directional selection on resource preference as long as the innate preference is away from the optimum. This argument predicts that the selective advantage of learning ability under directional selection should be transient-the advantage of learning disappears when the innate preference for the rewarding medium has approached 100\%. However, after almost 50 generations of selection, our flies were still far from this limit, and thus learning may still have helped 
to increase the proportion of eggs laid on the favored medium. Furthermore, slowing down of the response suggests that a $100 \%$ innate preference may be difficult or impossible to reach, in which case the advantage of learning may be permanent.

The ability to learn in the Learning Pineapple lines was much more strongly manifested when the flies were conditioned to avoid pineapple than when they were conditioned to avoid orange, even though this selection regime involved conditioning every generation to avoid orange. This strong asymmetry of the response to conditioning was specific to the Learning Pineapple selection regime. There was only a weak tendency for such asymmetry in the Learning Orange lines at generation 46, and it was not observed at all in another set of lines, which originated from the same base population and had been selected for improved response to conditioning in both directions (Mery and Kawecki 2002). Such an asymmetry would have been easier to explain if the flies had shown a strong innate preference for pineapple. If this had been the case, the flies would have avoided the orange medium during conditioning (in period 1) and would thus have had little opportunity to associate it with quinine. However, in the absence of conditioning the Learning Pineapple lines laid on average about $43 \%$ of eggs on the orange medium, which means that they should have had enough contact with the quinine-containing orange medium while being conditioned to avoid orange. One possibility is that under our assay conditions it is difficult for the flies to lay more than about $60 \%$ of eggs on the pineapple medium; this could be due to, for example, some sensory constraints and/or competition avoidance. Such a constraint would preclude a response to conditioning to avoid orange once the innate preference for pineapple had become close to $60 \%$. If so, then the response to conditioning in the Learning Pineapple lines might have been less asymmetric in the initial phase of the experiment. Without relevant data this remains a speculation.

It should also be noted that the observed response to conditioning is based on the proportion of eggs laid on the two media during six hours (i.e., two 3 -h periods) after the end of the conditioning period. Therefore, it is possible that the Learning Pineapple flies conditioned to avoid orange show a strong response immediately after conditioning, but quickly lose the response when they realize that the orange medium does not contain quinine any more. This process of erasing an old learned response through new experience is referred to as memory extinction (Bouton 1994). This idea is supported by the response observed within three hours (period 2) and between three and six hours (period 3) after conditioning. The proportion of eggs laid on the orange medium by Learning Pineapple flies conditioned to avoid orange was significantly smaller in period 2 than period 3 , and an effect of conditioning to avoid orange could only be detected in period 2. In contrast, the realized preference did not differ between the two periods for Learning Pineapple flies conditioned to avoid pineapple, as well as for those not conditioned. It is, however, not clear why the Learning Pineapple lines should show faster memory extinction when conditioned to avoid orange than when conditioned to avoid pineapple. Their selection regime favored flies that were not prone to memory extinction, that is, continued to avoid the orange medium three to six hours after the termination of conditioning. Thus, the asymmetry of the response of Learning Pineapple lines to conditioning remains unexplained.

Despite these open questions, our results directly demonstrate evolution of improved learning ability in a constant environment, in which the same behavioral response is always optimal.

\section{Effect of Learning on the Evolution of Innate Preference}

Comparison of the innate preference between the selection regimes Learning Orange and Innate Orange, as well as between Learning Pineapple and Innate Pineapple, indicates that the opportunity to learn has affected the evolution of innate preference, and that it did so differently in lines selected in different directions. Under selection favoring oviposition on the orange medium learning has slowed down the evolution of the innate preference: Innate Orange lines evolved a stronger innate preference for the rewarding medium (i.e., orange) than Learning Orange lines. In contrast, under selection for oviposition on pineapple learning has accelerated the evolution of the innate preference: Learning Pineapple lines evolved a stronger innate preference for their rewarding medium (i.e., pineapple) than Innate Pineapple lines. This synergism between the evolution of the innate preference and learning ability under the Learning Pineapple selection regime is also apparent in the pattern of variation among replicate selection lines. Across the replicate lines the learning score was positively correlated with the innate preference for pineapple, that is, the proportion of eggs laid on the pineapple medium in the absence of conditioning (Fig. 4 ). Despite involving only eight (generation 23) or four (generation 46) points, this correlation was close to significance. Although this correlation analysis should be regarded as exploratory, taken at face value it suggests that lines that evolved better learning responded more strongly to selection on the innate component of preference.

These results support the idea that learning may affect evolution of the innate components of resource preference, but do not unequivocally support either of the opposing notions that learning slows down evolution (Anderson 1995; Ancel 2000) versus that it accelerates it (Baldwin 1896; Hinton and Nowlan 1987; Maynard Smith 1987). Rather, it suggests that even in populations of common origin, learning may both hinder and facilitate the evolution of the innate component of preference, depending on the direction of selection. One way to view this problem is in terms of the effect of learning on the genotypephenotype map (Fig. 5). The following argument assumes that the power of learning to modify resource preference is limited; that is, that even with learning, the rewarding resource will not be $100 \%$ preferred, which seems a reasonable assumption, at least for invertebrates. If, for whatever reason, learning has a smaller effect on the realized preference (i.e., the phenotype) of individuals with stronger innate (genetic) preference for the rewarding resource, it will buffer the phenotypic expression of genetic differences in preference (Fig. 5a). This will tend to reduce the effective strength of selection on the genetic component of preference. In contrast, if within the population the effect of learning on realized preference is positively correlated with the innate preference for the rewarding resource, learning 
(a)

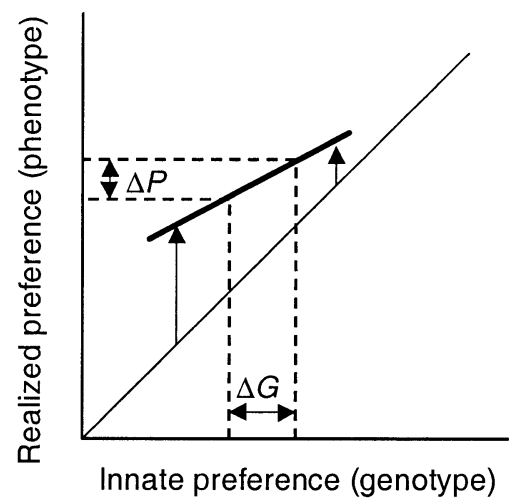

(b)

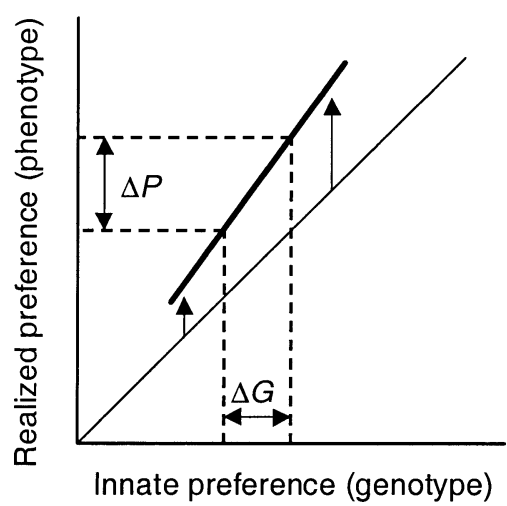

FIG. 5. Schematic representation of potential effects of learning on the genotype-phenotype map (light line: the genotype-phenotype map in the absence of learning; heavy line: the map as modified by learning). (a) If the shift of realized preference due to learning (symbolized by the arrows) is stronger in individuals that show weaker innate preference for the rewarding resource, learning will reduce the phenotypic effect $(\Delta P)$ of a given difference in innate preference $(\Delta G)$. (b) If the effect of learning on realized preference is positively correlated with the innate preference for the rewarding resource, the phenotypic effect of a difference in innate preference will be magnified.

will tend to magnify the phenotypic effects of genetic differences in preference (Fig. 5b). This will tend to amplify effective selection on the genetic component of preference. We have at present no data to test this hypothesis directly.

An alternative explanation for the greater evolutionary change of the innate preference of the Learning Pineapple than the Innate Pineapple lines invokes a potential additional selection pressure due to the exposure to quinine during conditioning (in period 1) in the former. The exposure to quinine may have had a detrimental effect on the number or quality of eggs laid in period 3 six hours later; these were the eggs used to breed the next generation. If so, Learning Pineapple flies would have been under stronger selection to avoid the orange medium than Innate Pineapple, because by avoiding the orange medium in period 1 the former would also avoid contact with quinine. However, the above argument would predict that replicate lines of the Learning Pineapple selection regime that have evolved a stronger innate preference for pineapple would have been under weaker selection for learning ability. This contradicts the positive correlation between innate preference for pineapple and the learning score observed among Learning Pineapple lines (Fig. 4). Furthermore, the same argument would hold for the selection to avoid pineapple in the Learning Orange lines, yet those lines evolved weaker innate preference for orange than the Innate Orange lines. One could still argue that Learning Orange flies were from the beginning less prone to this effect, as they experienced quinine in the pineapple medium, which from the start was the less preferred one. This would, however, not explain why the Learning Orange lines evolved a weaker innate preference for orange than the Innate Orange. Finally, we never observed any reduction of fertility or survival due to the exposure to quinine. Nonetheless, we cannot exclude that the exposure to quinine did contribute to the effective selection pressure on the innate components of oviposition substrate preference.

Finally, the outcome of selection may also have been affected by an interaction among innate preference, learning, and the flies' tendency for gregariousness. Drosophila melanogaster females like to oviposit in sites already containing conspecific eggs (Delsolar and Godoy 1971; Rockwell and Grossfield 1978). Thus, the proportion of eggs laid by an individual on each medium will depend on its innate preference and possibly learning, but also on the oviposition decisions of other individuals. This gregarious tendency might override the innate preference of an individual for pineapple if the rest of the population shows a strong preference for orange. If so, a rare allele for increased innate preference for pineapple would be effectively underexpressed, and the evolution of increased innate preference for pineapple would be constrained. This constraint would be alleviated if the rest of the population increased oviposition on pineapple in response to conditioning. This would thus be another mechanism in which learning could facilitate the evolution of innate preference. It would potentially explain both the weak response of the Innate Pineapple lines to selection on the innate component of preference, as well as the fast evolution of the innate component of preference in the Learning Pineapple lines. However, it is unclear whether the moderate preference for orange shown by all lines at the onset of the experimental evolution (58\% of eggs laid on the orange medium) was strong enough for the gregarious tendency to have a nonnegligible effect.

A common problem of all these scenarios with regard to the Learning Pineapple lines is the asymmetry of their response to conditioning, and specifically the apparent lack of a detectable response in period 3 to conditioning to avoid orange. In the absence of response to conditioning to avoid orange, learning would not be expressed in the course of selection in the Learning Pineapple selection regime, so it is hard to imagine how it should affect evolution of the innate preference. We see two potential solutions to this paradox. First, the overall mean response to conditioning may be too weak to be detected, but still have an appreciable effect on the evolution of the innate preference. For example, if only $10 \%$ of individuals showed a response to conditioning to avoid orange, the response would be hard to detect. Yet, if these were simultaneously the $10 \%$ with the strongest genetic preference for pineapple, the effective selection in favor of their genes would still be amplified. Second, as discussed above, the learning response of the Learning Pine- 
apple lines may have been less asymmetric in an early phase of the experiment when most of the evolutionary changes were taking place.

\section{Conclusion}

In this paper we have shown experimentally that learning ability can evolve even if the same behavioral response (the same resource choice) is always favored, contradicting the notion that learning should only be favored if the fitness payoffs of different responses vary between or within generations. We have also shown that, depending on the direction of selection, an opportunity to learn can either facilitate or hinder the evolutionary response of the innate component of preference. Although this result is difficult to interpret, it suggests that the solution of the long-standing controversy over the effect of learning on evolution may involve a synthesis of the two notions - that learning accelerates evolution versus that it slows it down-which have usually been discussed as contradictory.

\section{ACKNOWLEDGMENTS}

We thank N. Cueni, T. Flatt, and N. Vouilloz for help with the experiments, and V. Braithwaite, D. Ebert, O. Ronce, N. Vouilloz, and three anonymous referees for comments. This work was supported by Swiss National Science Foundation and Roche Research Foundation.

\section{Literature Cited}

Ackley, D., and M. Littman. 1991. Interactions between learning and evolution. Pp. 487-509 in C. Langton, C. Taylor, J. D. Farmer, and S. Rasmussen, eds. Artificial life II. Santa Fe Institute studies in the sciences of complexity. Vol. 10. AddisonWesley, Redwood City, CA.

Ancel, L. W. 1999. A quantitative model of the Simpson-Baldwin effect. J. Theor. Biol. 196:197-209.

—_ 2000. Undermining the Baldwin expediting effect: Does phenotypic plasticity accelerate evolution? Theor. Popul. Biol. 58:307-319.

Anderson, R. W. 1995. Learning and evolution: a quantitative genetics approach. J. Theor. Biol. 175:89-101.

Baldwin, J. M. 1896. A new factor in evolution. Am. Nat. 30: 441-451.

Belew, R. K. 1989. When both individuals and populations search: adding simple learning to the genetic algorithm. Pp. 34-41 in Proceedings of the third international conference on genetic algorithms, June 1989, Washington, DC.

Bouton, M. E. 1994. Conditioning, remembering and forgetting. J. Exp. Psychol. Anim. Behav. Processes 20:219-231.

Delsolar, E., and R. Godoy. 1970. Aggregation of preadult forms in D. melanogaster-changes due to prolonged oviposition. Arch. Biol. Med. Exp. 7:61-64.

Dukas, R. 1998. Evolutionary ecology of learning. Pp. 129-174 in R. Dukas, ed. Cognitive ecology. Univ. of Chicago Press, Chicago.

Fox, C. W. 1993. A quantitative genetic-analysis of oviposition preference and larval performance on two hosts in the bruchid beetle, Callosobruchus maculatus. Evolution 47:166-175.

French, R., and A. Messinger. 1994. Genes, phenes and the Baldwin effect. Pp. 277-282 in R. Brooks and P. Maes, eds. Artificial life IV. MIT Press, Cambridge, MA.

Futuyma, D. J., and G. Moreno. 1988. The evolution of ecological specialization. Annu. Rev. Ecol. Syst. 19:207-233.

Girvan, J. R., and V. A. Braithwaite. 1998. Population differences in spatial learning in three-spined sticklebacks. Proc. R. Soc. Lond. B 265:913-918.

Gould-Beierle, K. L., and A. C. Kamil. 1998. Use of landmarks in three species of food-storing corvids. Ethology 104:361-377.

Hinton, G. E., and S. J. Nowlan. 1987. How learning can guide evolution. Complex Syst. 1:495-502.

Jackson, R. R., and C. M. Carter. 2001. Geographic variation in reliance on trial-and-error signal derivation by Portia labiata, an araneophagic jumping spider from the Philippines. J. Insect Behav. 14:799-827.

Johnston, T. D. 1982. Selective costs and benefits in the evolution of learning. Adv. Study Behav. 12:65-106.

Laverty, T. M., and R. C. Plowright. 1988. Flower handling by bumblebees: a comparison of specialists and generalists. Anim. Behav. 36:733-740.

Littell, R. C., R. J. Freund, and P. C. Spector. 1991. SAS system for linear models. 3rd. ed. SAS Institute, Cary, NC.

Mayley, G. 1996. Landscapes, learning costs and genetic assimilation. Evol. Comput. 4:213-234.

Maynard-Smith, J. 1987. When learning guides evolution. Nature 329:761-762.

McNeely, C., and M. C. Singer. 2001. Contrasting the roles of learning in butterflies foraging for nectar and oviposition sites. Anim. Behav. 61:847-852.

Mery, F., and T. J. Kawecki. 2002. Experimental evo lution of learning ability in fruit flies. Proc. Natl. Acad. Sci. USA 99: $14274-14279$.

- 2003. A fitness cost of learning ability in Drosophila melanogaster. Proc. R. Soc. Lond. B 270:2465-2469.

Morgan, C. L. 1896. On modification and variation. Science 4: $733-740$.

Nosil, P. 2002. Transition rates between specialization and generalization in phytophagous insects. Evolution 56:1701-1706.

Osborn, H. F. 1896. Ontogenic and phylogenic variation. Science 4:786-789.

Papaj, D. R. 1994. Optimizing learning and its effect on evolutionary change in behavior. Pp. 133-154 in Behavioral mechanisms in evolutionary biology. Univ. of Chicago Press, Chicago.

Pigliucci, M., and C. J. Murren. 2003. Genetic assimilation and a possible evolutionary paradox: Can macroevolution sometimes be so fast as to pass us by? Evolution 57:1455-1464.

Radtkey, R. R., and M. C. Singer. 1995. Repeated reversals of hostpreference evolution in a specialist insect herbivore. Evolution 49:351-359.

Robinson, B. W., and R. Dukas. 1999. The influence of phenotypic modifications on evolution: the Baldwin effect and modern perspectives. Oikos 85:582-589.

Rockwell, R. F., and J. Grossfield. 1978. Drosophila-behavioral cues for oviposition. Am. Midl. Nat. 99:361-368.

Scriber, J. M. 2002. Evolution of insect-plant relationships: chemical constraints, coadaptation, and concordance of insect/plant traits. Entomol. Exp. Appl. 104:217-235.

Singer, M. C., C. D. Thomas, and C. Parmesan. 1993. Rapid humaninduced evolution of insect host associations. Nature 366: $681-683$.

Stephens, D. 1991. Change, regularity, and value in the evolution of animal learning. Behav. Ecol. 2:77-89.

Sullivan, K. A. 1988. Age-specific profitability and prey choice. Anim. Behav. 36:613-615.

Thompson, J. N. 1993. Preference hierarchies and the origin of geographic specialization in host use in swallowtail butterflies. Evolution 47:1585-1594.

- 1998. The evolution of diet breadth: monophagy and polyphagy in swallowtail butterflies. J. Evol. Biol. 11:563-578.

Wasserman, S. S. 1986. Genetic variation in adaptation to foodplants among populations of the southern cowpea weevil, Callosobruchus maculatus-evolution of oviposition preference. Entomol. Exp. Appl. 42:201-212.

Wehling, W. F., and J. N. Thompson. 1997. Evolutionary conservatism of oviposition preference in a widespread polyphagous insect herbivore, Papilio zelicaon. Oecologia 111:209-215.

Corresponding Editor: J. Merilä 